\title{
Overhead conductor monitoring system for the evaluation of the low sag behavior
}

\author{
I. Albizu, E. Fernández, A. J. Mazón, Member, IEEE, M. Bedialauneta, and K. Sagastabeitia
}

\begin{abstract}
The paper describes a monitoring system for the evaluation of the low sag behavior of the overhead conductors in operating lines. The monitoring system measures the conductor tension and temperature and it also measures the wind speed to evaluate the load in the conductor due to wind. This system includes a methodology for the analysis of the measured data and the comparison of these data with the theoretical values obtained by mechanical calculation.
\end{abstract}

Index Terms-HTLS conductor, monitoring system, low sag characterization, overhead line

\section{INTRODUCTION}

The conductor replacement by high temperature low sag (HTLS) conductors allows increasing the line power flow securely and safely without the need to strengthen the towers [1]. These conductors work at higher temperatures than the conventional conductors and their coefficients of thermal expansion (CTE) are lower than the CTE of the conventional conductor [2-8].

Low sag conductors are based on the conductor behavior above the knee-point temperature, where the aluminum is slack and only the core is under tension. This occurs due to the higher CTE of the aluminum. Hence, above the knee-point temperature the behavior of the conductor is based on the CTE of the core, which is lower than the CTE of the conductor. For this reason, the sag increase with temperature is lower above the knee-point temperature (Fig. 1).

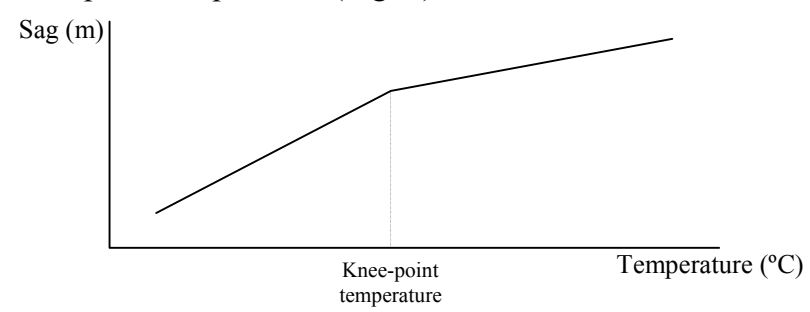

Fig. 1. Knee-point temperature

This work is financially supported by the University of the Basque Country UPV/EHU under the project EHU09/18, the Ministerio de Ciencia e Innovación under the project DPI2009-08454, and the Eusko Jaurlaritzako Hezkuntza, Unibertsitate eta Ikerketa Saila (Euskal unibertsitate-sistemako ikerketa-taldeak Ref. IT532-10)

The authors are with the Electrical Engineering Department, Faculty of Engineering of Bilbao, University of the Basque Country, Bilbao 48013, Spain (e-mail: igor.albizu@ehu.es; elvira.fernandezh@ehu.es; javier.mazon@ehu.es; miren.bedialauneta@ehu.es; koldo.sagastabeitia@ehu.es)
The tests carried out by the manufacturers do not reproduce the actual operation conditions. Therefore, to validate the conductor characteristics given by the manufacturer, the systems that monitor the conductor in operation are useful.

The authors have developed a monitoring system for the evaluation of the low sag behavior of the overhead conductors. The paper describes this system.

\section{EVALUATION OF HTLS CONDUCTORS IN OPERATION}

One of the main characteristics to be verified in the operation of the HTLS conductors is the low sag behavior. This is achieved comparing the conductor theoretical tensiontemperature behavior with the measured values. Figure 2 shows some examples of results that could be obtained. In the example a) there is good correspondence between the expected theoretical values and the measured ones. In the example b) the measured knee-point temperature $\left(70^{\circ} \mathrm{C}\right)$ is higher than the expected one $\left(40^{\circ} \mathrm{C}\right)$.
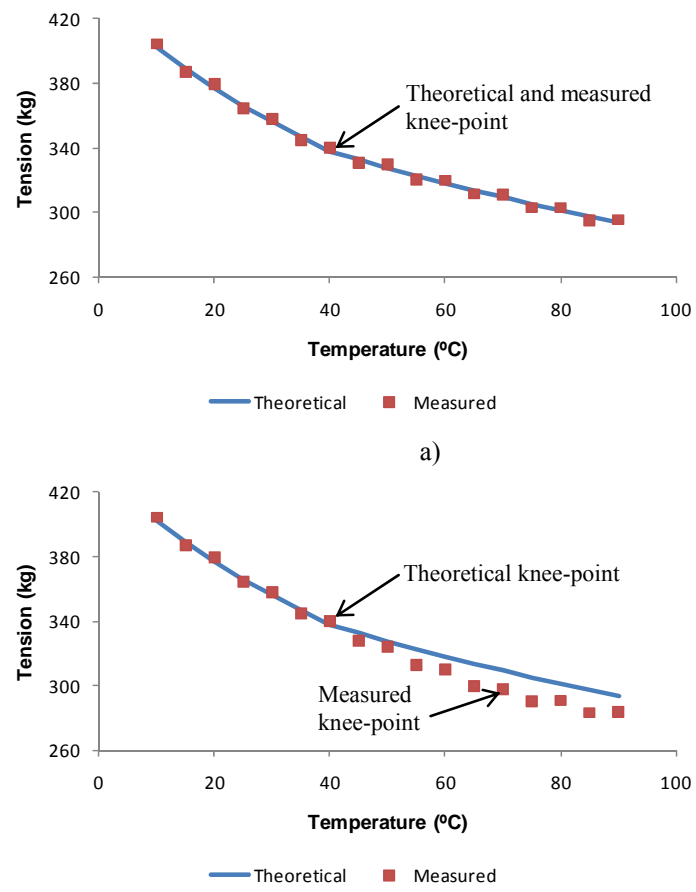

b)

Fig. 2. Theoretical tension-temperature curve and measured values

a) Correspondence between theoretical and measured values

b) Measured knee-point temperature higher than expected

To compare the theoretical values with the measured values, the measured tension-temperature curve should 
represent correctly the actual behavior of the conductor. This is not trivial because some factors can distort the curve as it is described below.

In a similar way, the calculated theoretical curve should represent correctly the actual behavior of the conductor. For this purpose, different sag-tension calculation methods have been analyzed so that the method used for the calculation is suitable for the characteristics of the conductor.

There are two types of outdoor testing which are useful for the qualification and acceptation of HTLS conductors: the outdoor laboratory tests and the operating line tests [9]. In an outdoor laboratory test the conductor is at low voltage and the injected current can be controlled whereas in an operating line test the conductor is at the full line voltage and the current through the line depends on the variation of the load. The operating line test is useful in evaluating potential installation difficulties, corrosion and corona issues under outdoor conditions and in testing proposed installation guidelines and maintenance rules whereas the outdoor laboratory test is useful in measuring the sag, the tension and the conductor temperature under sustained high current conditions and naturally varying weather conditions.

Some examples of operating lines tests are shown in [1013]. In [10] a project where different types of HTLS conductors are installed in several lines is presented. The sag, the tension, the current and the weather magnitudes are measured every 10 minutes. Besides, the splice resistance, the conductor and hardware temperature, the corona, the EMF and the visual appearance are evaluated in periodical site visits. The vibration is also recorded and downloaded during site visits. One of the lines of the project presented in [10] is described in [11]. In this case, gap-type and invar core conductors are monitored. In [12] an ACCR conductor is monitored. The tension and the weather magnitudes are measured every 10 minutes. One of the main drawbacks of the operating line tests is observed in this case. This is the difficulty to obtain high temperature data because of the dependence on the line load. In [13], another example of the monitoring of an ACCR conductor is shown. In this case, the conductor temperature and the weather magnitudes are measured.

Some outdoor laboratory tests carried out for the evaluation of the low sag behavior are shown in [14-18]. In [14], the knee-point temperature evaluation of an invar core conductor is shown. In [15], the tension and the temperature of a gaptype conductor, the current intensity and the weather magnitudes are measured. The obtained CTE above the kneepoint temperature is higher than the theoretical value. In [16], the temperature of an ACCR conductor is measured with 25 thermocouples. The tension and the ambient temperature are also measured. In [17], the tension-temperature curve of an ACCR conductor is obtained up to $300{ }^{\circ} \mathrm{C}$. Thermal hysteresis of the aluminum is observed. This effect is also observed in [18] where the temperature of an ACSR conductor is increased in steps to $60,80,100,120,140$ and $150{ }^{\circ} \mathrm{C}$ and held at each level for one hour. Then, the temperature is decreased with the same steps. Sags with increasing temperatures are less than sags with decreasing temperatures.

Below the main aspects of the developed monitoring system for the evaluation of the conductor low sag behavior are described.

\section{THEORETICAL BEHAVIOR}

As it has been mentioned before, the calculated theoretical tension-temperature curve should represent correctly the actual behavior of the conductor. Thus, the behavior of the core and the aluminum as a function of the elastic modulus and CTE should be modeled. The knee-point temperature where the aluminum gets slack should be modeled too. The calculation method used to model the conductor behavior is adapted from sag-tension method developed by some of the authors taking into account the requirements of the HTLS conductors $[19,20]$. This sag-tension model is simplified for the evaluation of the low sag behavior. Thus, the knee-point temperature is defined and a different conductor behavior is considered above and below this temperature. Below the knee-point temperature the conductor area, elastic modulus and coefficient of expansion values are taken, and above the core values. The creep deformation is assumed to be constant. As a result, the parameters included in the sag-tension model are the following:

- Span length: $s$

- Conductor weight: $\omega_{\text {con }}$

- Elastic modulus of the conductor and the core: $E_{c o n}$, Ecore

- Area of the conductor and the core: $A_{\text {con }}, A_{\text {core }}$

- Coefficient of expansion of the conductor and the core: $\alpha_{\text {con }}, \alpha_{\text {core }}$

- Knee-point temperature: $\theta_{\text {knee }}$

The model also needs a tension-temperature reference $T_{o^{-}}$ $\theta_{\mathrm{o}}$. This is obtained from measured values at the beginning of the period of analysis.

The first step is the calculation of the conductor reference length $L_{o}$, which is the span length at the reference tension $T_{o}$ and temperature $\theta_{0}$. The conductor weight in the reference condition is taken into account $\omega_{0}$. This is usually the conductor weight $\omega_{\text {con }}$ if no overload is present.

$$
\begin{aligned}
c_{o} & =\frac{T_{o}}{\omega_{o}} \\
L_{o} & =2 \cdot c_{o} \cdot \sinh \left(\frac{s / 2}{c_{o}}\right)
\end{aligned}
$$

The next step is the calculation of the tensión $T_{k n e e}$ at the knee-point temperature $\theta_{\text {knee }}$. For this purpose, the tension value is iterated until the catenary length $L_{c a t}$ and the conductor length $L_{c o n}$ have the same values. The conductor weight in the knee-point condition is taken into account $\omega_{\text {knee }}$. This is usually the conductor weight $\omega_{\text {con }}$ if no overload is 
present. The equation (5) assumes that the reference temperature $\theta_{0}$ is lower than the knee-point temperature $\theta_{\text {knee }}$, which is usually the case.

$$
\begin{aligned}
& c_{\text {knee }}=\frac{T_{\text {knee }}}{\omega_{\text {knee }}} \\
& L_{\text {cat }}=2 \cdot c_{\text {knee }} \cdot \sinh \left(\frac{s / 2}{c_{\text {knee }}}\right) \\
& L_{\text {con }}=L_{o} \cdot\left(1+\alpha_{\text {con }} \cdot\left(\theta_{\text {knee }}-\theta_{o}\right)+\frac{T_{\text {knee }}-T_{o}}{E_{\text {con }} \cdot A_{\text {con }}}\right)
\end{aligned}
$$

Finally, the $T$ tension values are obtained from the $\theta$ temperature values of the temperature period of the analysis. For this purpose, for each temperature value the tension value is iterated until the catenary length $L_{c a t}$ and the conductor length $L_{c o n}$ have the same values. The conductor weight is taken into account $\omega$. This is usually the conductor weight $\omega_{\text {con }}$ if no overload is present. The conductor length is given by (8) if the temperature is below the knee-point temperature and it is given by (9) if it is above. The equations (8) and (9) assume that the reference temperature $\theta_{\mathrm{o}}$ is lower than the knee-point temperature $\theta_{\text {knee, }}$ which is usually the case.

$$
\begin{aligned}
& c=\frac{T}{\omega} \\
& L_{c a t}=2 \cdot c \cdot \sinh \left(\frac{s / 2}{c}\right) \\
& L_{\text {con }}=L_{o} \cdot\left(1+\alpha_{\text {con }} \cdot\left(\theta-\theta_{o}\right)+\frac{T-T_{o}}{E_{\text {con }} \cdot A_{\text {con }}}\right) \\
& L_{\text {con }}=L_{o} \cdot\left(1+\alpha_{\text {con }} \cdot\left(\theta_{\text {knee }}-\theta_{o}\right)+\alpha_{\text {core }} \cdot\left(\theta-\theta_{\text {knee }}\right)+\frac{T_{\text {knee }}-T_{o}}{E_{\text {con }} \cdot A_{\text {con }}}+\frac{T-T_{\text {knee }}}{E_{\text {core }} \cdot A_{\text {core }}}\right)
\end{aligned}
$$

\section{MONITORING SYSTEM}

The developed monitoring system measures the conductor tension and temperature and it also measures the wind speed to evaluate the conductor load due to wind.

Figure 3 shows the conductor tension and temperature values measured in a line in operation during a whole day. The direct relation between the tension and the temperature is obvious. The tension value increases when the temperature value decreases and vice versa.
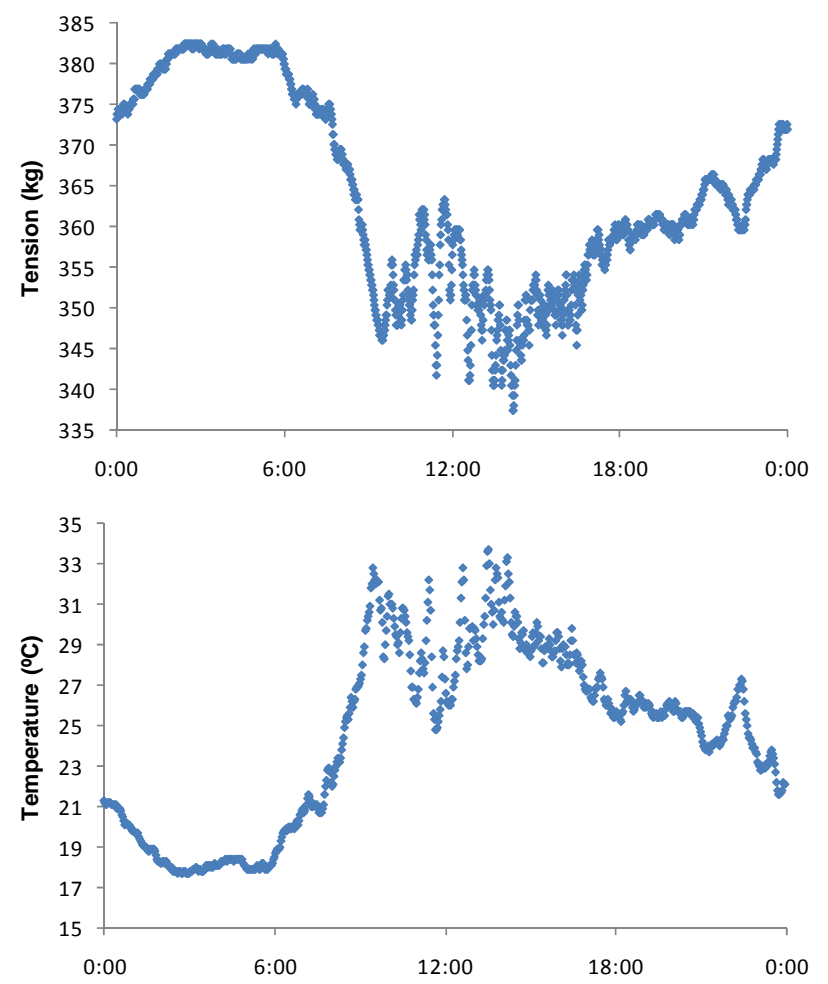

Fig. 3. Conductor tension and temperature measured in a line in operation

\section{A. Monitoring difficulties}

There are some difficulties obtaining the tensiontemperature values that represent the actual behavior of the conductor in operation. These are described below.

1) Synchronization of the tension and temperature measurements

The commercial systems that monitor the tension and the temperature are separate systems that measure the data independently. Hence, the instants that measurements are acquired should be synchronized as far as possible.

2) Measurement fluctuations

When the weather conditions and the current intensity do not vary their values the conductor temperature and tension values do not change their values either. However, as the weather conditions and the current intensity can change considerably with time, in this case there are fluctuations in the conductor and tension values. Besides, the tension also fluctuates due to the conductor vibration caused by the wind.

\section{3) Wind load}

The conductor load increases due to the pressure that the wind applies on the conductor surface. Hence, the wind changes the conductor tension at a given conductor temperature.

\section{B. Data processing}

The tension-temperature values that represent the actual behavior of the conductor in operation are obtained processing the acquired measurements. The synchronization of the measurements, the fluctuation of the measurements and the effect of the wind are taken into account.

To minimize the effect of the fluctuations of the measured 
values, the measurements are valid for the analysis of the conductor behavior only if these fluctuations are low. For this purpose, the standard deviation of the measurements is calculated during a defined period of time and if the deviation is below a threshold value the period is valid. If the period is valid the mean values of the measurements are calculated and the results are recorded in the database of the tensiontemperature values that represent the behavior of the conductor. Figure 4 shows the standard deviation of the measurements (Fig. 3) during a period of time of 30 minutes.
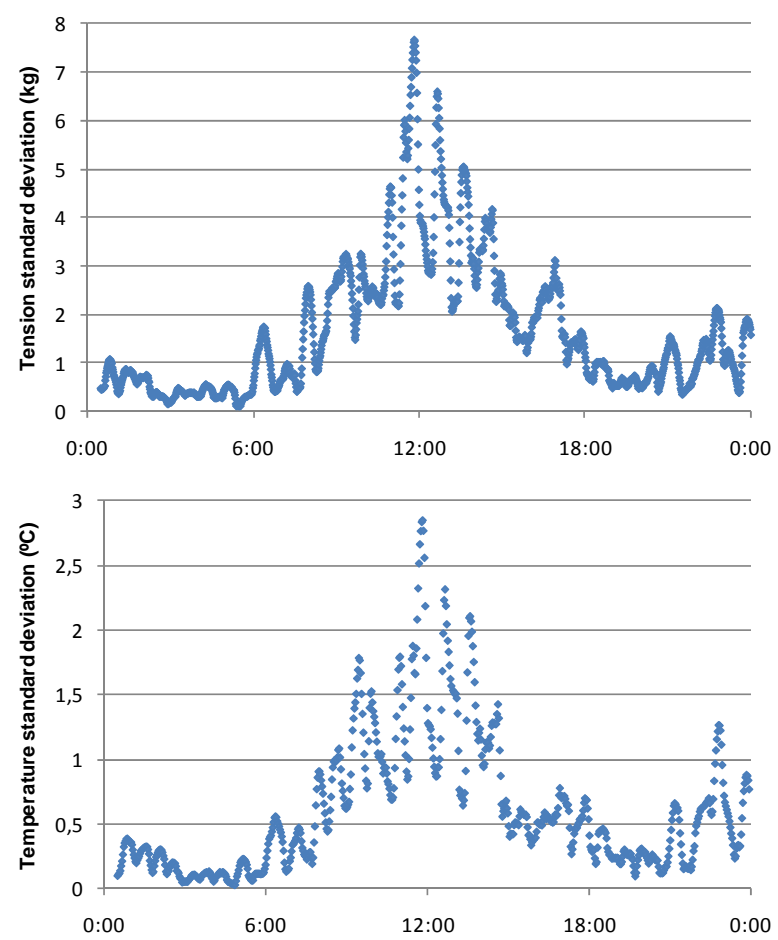

Fig. 4. Standard deviation of the tension and the temperature

Figure 5 shows the tension-temperature values that will be part of the database when a threshold value of $0.5 \mathrm{~kg}$ is taken for the tension standard deviation in the case of the example given in Figure 3. When this is applied to longer datasets, the obtained values are within a larger range of tension and temperature values.

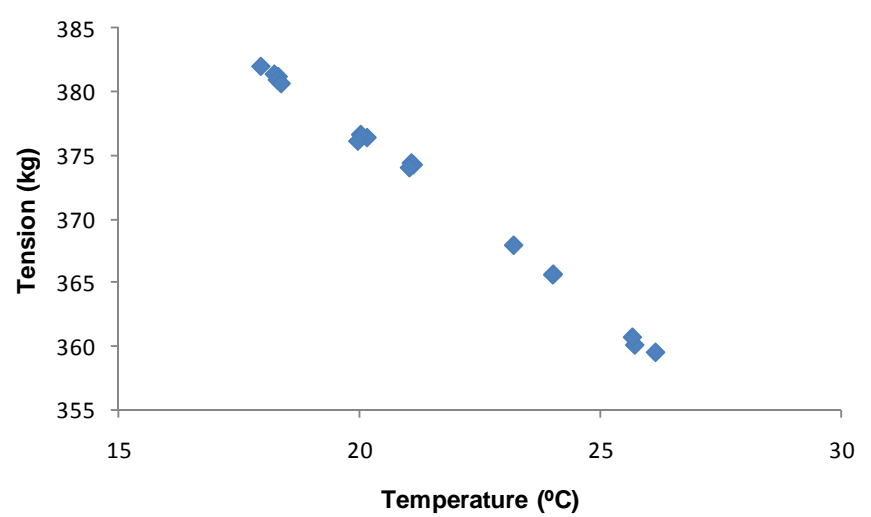

Fig. 5. Tension-temperature values for the database
Figure 6 shows the theoretical tension-temperature curve of the conductor monitored in the presented example. The values obtained for the database in the example (Fig. 5) are also included. A correspondence between the theoretical and measured values is observed. However, a larger range of measured values is needed to obtain conclusions of the conductor behavior.

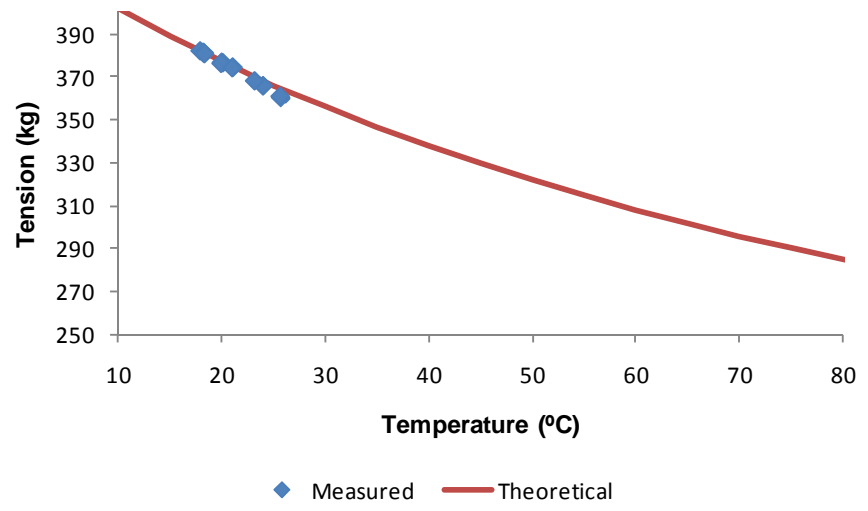

Fig. 6. Theoretical and measured tension-temperature values

Figure 7 shows the measurements obtained for several months. These values have been obtained using a period of time of 40 minutes and different tension deviation threshold values $(1.5 \mathrm{~kg}, 0.7 \mathrm{~kg}$ and $0.3 \mathrm{~kg})$. Figure 8 shows the number of measurements obtained for the different tension deviation threshold values. It is observed that the higher the tension deviation threshold is, the higher the number of obtained measurements. However, the dispersion of the obtained measurements is also higher. When the threshold decreases, the dispersion is reduced but decreasing the number of measurements and the range of the obtained values. Figure 8 clearly shows how if the threshold decreases its value the measurements above $25^{\circ} \mathrm{C}$ are lost.

Apart from the tension deviation threshold, the value of the period also affects the obtained measurements. The longer is the period it is more difficult that the deviation is below the threshold value. This is shown comparing the Figure 9, where the period is 60 minutes and the Figure 7.b) the period is 40 minutes. In both Figures the tension deviation threshold is 0.7 $\mathrm{kg}$. If the obtained values are stable for a longer period they give a higher confidence. Hence, the measurement quality is higher with long periods and low threshold values, but with a lower number of measurements and a smaller range of values. 

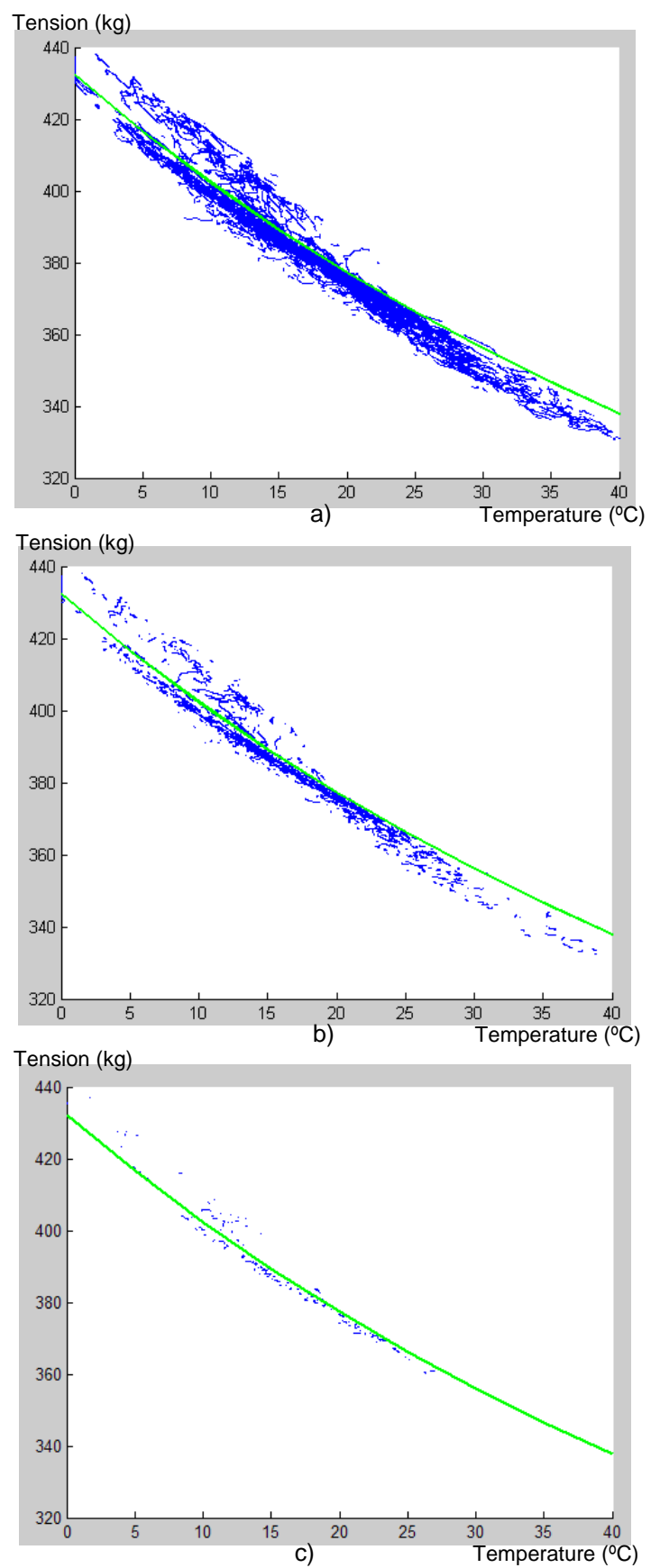

Fig. 7. Theoretical and measured tension-temperature values. Period of time: 40 minutes
a) Deviation threshold: $1.5 \mathrm{~kg}$
b) Deviation threshold: $0.7 \mathrm{~kg}$
c) Deviation threshold: $0.3 \mathrm{~kg}$
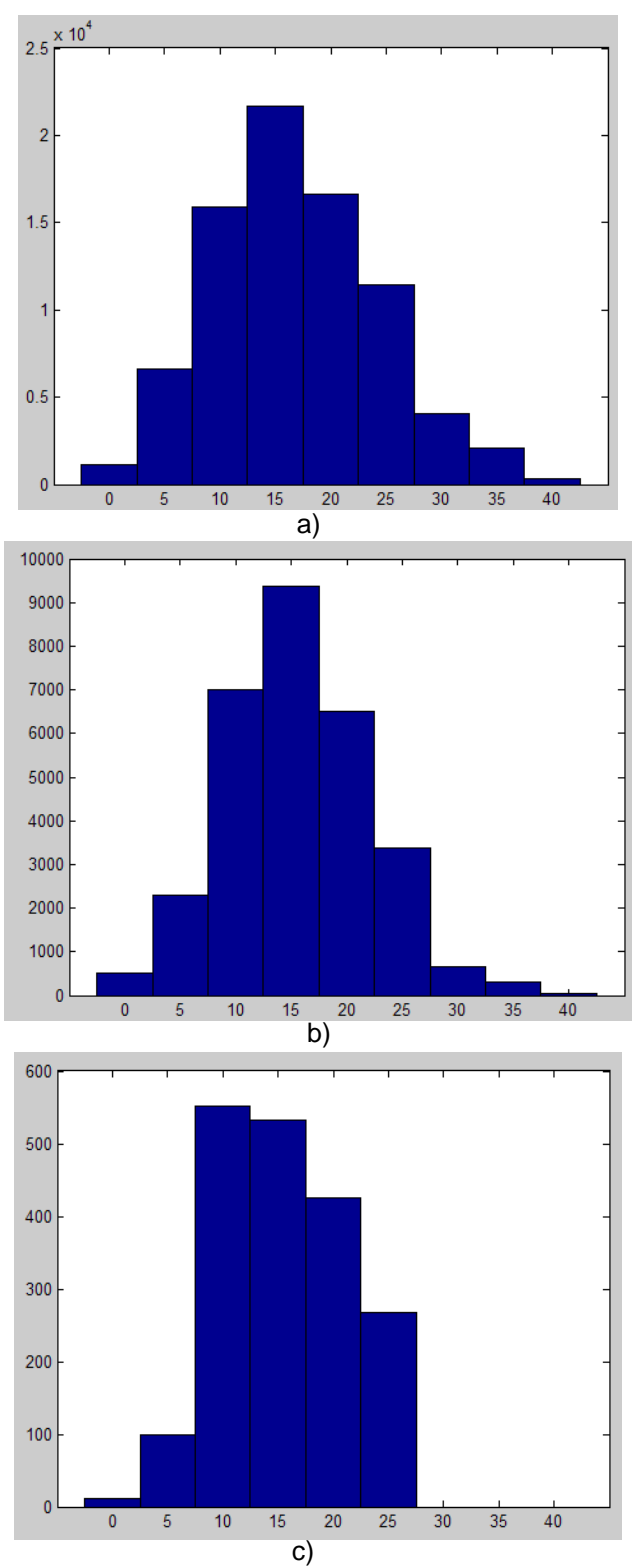

Fig. 8. Number of tension-temperature values. Period of time: 40 minutes a) Deviation threshold: $1.5 \mathrm{~kg}$

b) Deviation threshold: $0.7 \mathrm{~kg}$

c) Deviation threshold: $0.3 \mathrm{~kg}$ 


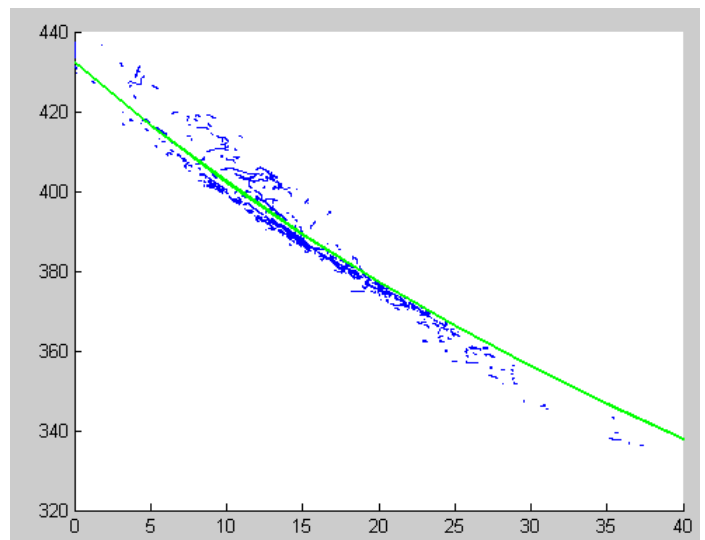

Fig. 9. Theoretical and measured tension-temperature values. Period of time: 60 minutes. Deviation threshold: $0.7 \mathrm{~kg}$

With respect to the evaluation of the conductor, that is to say the comparison between the measurements and the theoretical curve, a correspondence is observed although there are some differences. The measured tension at high temperatures is slightly lower than expected. Anyway, before obtaining any final conclusion a deeper analysis of the measurements could be carried out.

\section{Conclusions}

The monitoring system for the characterization of the low sag behavior of the overhead conductors developed by the authors has been described. This system includes a methodology for the analysis of the measured data.

The sag-tension model used for the evaluation of the low sag behavior has been described in detail. The knee-point temperature is defined and a different conductor behavior is considered above and below this temperature. Below the knee-point temperature the conductor parameter values are taken, and above the core values.

The monitoring and the analysis of the measured data have also been described. From measurements obtained for several months the influence of the period of time and the tension deviation threshold has been analyzed. The measurement quality is higher with long periods and low threshold values, but with a lower number of measurements and a smaller range of values.

Finally, the convenience of a deeper analysis of the measurements has been indicated. For example, the influence of the wind could be analyzed.

\section{ACKNOWLEDGMENT}

The authors gratefully acknowledge the support of the Iberdrola utility for the help with the achievement of this project.

\section{REFERENCES}

[1] I. Albizu, A. J. Mazon, and I. Zamora, "Methods for increasing the rating of overhead lines", IEEE PowerTech, St. Petersburg, 2005.

[2] CIGRE Brochure 244, "Conductors for the Uprating of Overhead Lines", 2004
[3] K. Yonezawa, K. Kinoshita, "Gap type conductor" presented at the IEEE TP \& C Line Design Meeting, Las Vegas, USA, 2005.

[4] M. Landeira, P. Morentin, A.J. Mazon, and I. Albizu, "The high temperature cable solution for electrical overhead distribution lines", DYNA, Vol. 82, No. 5, pp. 226-230, 2007.

[5] F.R. Thrash, "ACSS" presented at the IEEE TP \& C Line Design Meeting, Albuquerque, USA, 2006.

[6] S. Sasaki, T. Takebe, K. Miyazaki, M. Yokota, K. Sato, S. Yoshida, and I. Matsubara, "ZTACIR New Extra-Heat Resistant Galvanized InvarReinforced Aluminum Alloy Conductor", Sumitomo Electric Technical Review, Vol. 24, pp. 117-123, 1985.

[7] C. McCullough, "Update on ACCR conductor", presented at the IEEE TP \& C Line Design Meeting, Albuquerque, USA, 2006.

[8] D. Bryant, "ACCC conductor update", presented at the IEEE TP \& C Line Design Meeting, Orlando, USA, 2007.

[9] CIGRE Brochure 426, "Guide for qualifying high temperature conductors for use on overhead transmission lines", 2010

[10] B. Clairmont, "High-temperature low-sag transmission conductors", EPRI, 2008.

[11] A. Goel, "Hydro One Networks", IEEE TP \& C Line Design Meeting, Las Vegas-USA, 2005.

[12] L. Custer, "DOE-3M Demonstration Project", IEEE TP \& C Line Design Meeting, Albuquerque-USA, 2006.

[13] S. Kupke, "Pilot project-High temperature low sag conductors", CIGRE WG B2.42, Stockholm-Sweden, 2010

[14] S.Y. Park, "Reconductoring case study using STACIR conductor", IEEE TP \& C Line Design Meeting, Las Vegas-USA, 2005.

[15] S. P. Hoffmann, "The "Matthew" GZTACSR ("Gap-Type") conductor", IEEE TP \& C Line Design Meeting, Las Vegas-USA, 2005.

[16] T. Seppa, "Variability of conductor temperature in a two span high temperature test line at ORNL", IEEE TP \& C Line Design Meeting, Las Vegas-USA, 2005.

[17] C. McCullough, "Update on ACCR conductor", IEEE TP \& C Line Design Meeting, Albuquerque-USA, 2006.

[18] T. Seppa, D. Stoddart, "Sag calculation errors at high temperatures", IEEE Conductors Meeting, Minneapolis-USA, 2010

[19] I. Albizu, A. J. Mazon, and I. Zamora, "Flexible strain-tension calculation method for gap-type overhead conductors", IEEE Trans. on Power Del., Vol. 24, No. 3, pp. 1529-1537, 2009.

[20] I. Albizu, A. J. Mazon, V. Valverde, and G. Buigues,: “Aspects to take into account in the application of mechanical calculation to high temperature low sag conductors", IET Gener. Transm. Distrib., Vol. 4, No. 5, pp. 631-640, 2010. 